Analele Universităţii de Vest, Timişoara

Seria Matematică - Informatică

LIII, 1, (2015), 57- 76

\title{
Existence of three positive solutions for boundary value problem with fractional order and infinite delay
}

Hedia Benaouda

\begin{abstract}
In this paper we investigate the existence three positives solutions by using Leggett-Williams fixed point theorem in cones for three boundary value problem with fractional order and infinite delay.
\end{abstract}

AMS Subject Classification (2000). 26A33, 34B15.

Keywords. differential equations, Riemann-Liouville fractional derivative, fractional integral, positive solutions, Leggett-Williams fixed-point theorem, infinite delay, three boundary value problem.

\section{Introduction}

This paper is concerned with the existence of three positive solutions for three boundary value problems (BVP for short) of fractional differential equations with infinite delay. We consider the BVP of the form :

$$
\begin{gathered}
D^{\alpha} y(t)=f\left(t, y_{t}\right), \text { a.e, } t \in J=[0,1], \alpha \in(2,3] \\
y(0)=y^{\prime}(0)=0, \beta y(\eta)=y(1) \\
y(t)=\phi(t), \quad t \in(-\infty, 0]
\end{gathered}
$$


where $D^{\alpha}$ is the Riemann-Liouville fractional derivative, $\beta>0, \eta \in(0,1)$ such that $0<\beta \eta^{\alpha-1}<1, f: J \times \mathcal{B} \rightarrow[0,+\infty)$ is a given function satisfying some assumptions that will be specified later, $\phi \in \mathcal{B}$ and $\mathcal{B}$ is a phase space that will defined in section 2. For any function $y$ and any $t \in[0,1]$, we denote by $y_{t}$ the element of $\mathcal{B}$ defined by $y_{t}(\theta)=y(t+\theta)$ for $\theta \in(-\infty, 0]$. We assume that the histories $y_{t}$ belong to $\mathcal{B}$.

The notion of the phase space $\mathcal{B}$ plays an important role in the study of both qualitative and quantitative theory for functional differential equations. A usual choice is a seminormed space satisfying suitable axioms, which was introduced by Hale and Kato [12] (see also Kappel and Schappacher [14] and Schumacher [27]. For a detailed discussion on this topic we refer the reader to the book by [13].

Fractional differential equations have been of great interest recently. This is because of both the intensive development of the theory of fractional calculus itself and the applications of such constructions in various scientific fields such as physics, mechanics, chemistry, engineering, etc. For details,see $[7,15-18,20,22]$ and the references therein.

The solution of differential equations with fractional order is quite involved. Therefore, the theory of fractional differential equations has been developed very quickly and the investigation for the existence of its solutions has attracted a considerable attention from researches . (see $[2,4,5,8,16,22]$ and the references therein).

In recent years, many researchers focused on the solutions, especially the positive solutions of fractional differential equation boundary value problems, we refer to $[1,3,6,21,26,28,29]$ and references therein.

Some analytical methods are presented, such as the popular Laplace transform method [25], the Fourier transform method [23], the iteration method and the Green function method. Numerical schemes for solving fractional differential equations are introduced, for example, in [24].

Our results complement the few results devoted to existence of positive solutions for functional differential equations using different method krasnoselskii fixed point theorem, index fixed point theorem. To our best Knowledge, there are few results for existence of positive solutions for functional differential equations with fractional order and using Legett Williams fixed point theorem. Also, as far as we know, no papers exist in the literature devoted to such problems with existence of positive solutions for functional differential equations with fractional order and infinite delay.

Over the past several years it has become apparent that existence of positive solutions for differential equations with delay arise also in several 
Vol. LIII (2015) Existence of three positive solutions for boundary value problem 59

areas such as in classical electrodynamics, in population models, in models of commodity price fluctuations, and in models of blood cell productions. they also arise from the modeling of infection disease transmission, the modeling of immune response systems and the modeling of respiration, where the delay is due to the time required to accumulate an appropriate dosage of infection or antigen concentration.

\section{Preliminaries}

In this section, we introduce notations, definitions, and preliminary facts which are used throughout this paper. By $C(J, R)$ we denote the Banach space of all continuous functions from $J$ into $R$ with the norm:

$$
\|y\|_{\infty}:=\sup \{|y(t)|: t \in J\} .
$$

For the definition of the phase space $\mathcal{B}$ we introduce the following axioms.

$\left(A_{1}\right)$ If $y:(-\infty, 1) \rightarrow R,, y_{0} \in \mathcal{B}$, then for every $t \in[0,1]$ the following conditions hold:

(i) $y_{t} \in \mathcal{B}$,

(ii) There exists a positive constant $H$ such that $|y(t)| \leq H\left\|y_{t}\right\|_{\mathcal{B}}$;

(iii) There exist two functions $K(\cdot), M(\cdot): R_{+} \rightarrow R_{+}$, independent of $y$, with $K$ continuous and $M$ locally bounded such that:

$$
\left\|y_{t}\right\|_{\mathcal{B}} \leq K(t) \sup \{|y(s)|: 0 \leq s \leq t\}+M(t)\left\|y_{0}\right\|_{\mathcal{B}} .
$$

$\left(A_{2}\right)$ For the function $y($.$) in \left(A_{1}\right), y_{t}$ is a $\mathcal{B}$-valued continuous function on $[0,1]$.

$\left(A_{3}\right)$ The space $\mathcal{B}$ is complete.

Denote

$$
K=\sup \{K(t): t \in[0,1]\}
$$

and

$$
M=\sup \{M(t): t \in[0,1]\} .
$$

Definition 2.1. ([10,?ElSayed9]). The fractional (arbitrary) order integral of the function $h \in L^{1}\left([a, b], R_{+}\right)$of order $\alpha \in R_{+}$is defined by:

$$
I_{a}^{\alpha} h(t)=\int_{a}^{t} \frac{(t-s)^{\alpha-1}}{\Gamma(\alpha)} h(s) d s
$$


where $\Gamma$ is the gamma function. When $a=0$, we write $I^{\alpha} h(t)=h(t) * \varphi_{\alpha}(t)$, where $\varphi_{\alpha}(t)=\frac{t^{\alpha-1}}{\Gamma(\alpha)}$ for $t>0$, and $\varphi_{\alpha}(t)=0$ for $t \leq 0$, and $\varphi_{\alpha} \rightarrow \delta(t)$ as $\alpha \rightarrow 0$, where $\delta$ is the delta function.

Definition 2.2. ([10, ?ElSayed9]). For a function $h$ given on the interval $[a, b]$, the $\alpha$ th Riemann-Liouville fractional-order derivative of $h, \alpha \in R_{+}$is defined by:

$$
D_{0^{+}}^{\alpha} h(t)=\frac{1}{\Gamma(n-\alpha)}\left(\frac{d}{d t}\right)^{n} \int_{0}^{t}(t-s)^{n-\alpha-1} h(s) d s .
$$

Let $(E,\|\|$.$) be a Banach space and C \subset E$ be a cone in $E$. by a concave, positive and continuous functional $\psi$ on $C$, we mean a continuous mapping

$$
\psi: C \rightarrow[0, \infty)
$$

with

$$
\psi(\lambda x+(1-\lambda) y) \geq \lambda \psi(x)+(1-\lambda) \psi(y) \text { for all } x, y \in C \text { and } \lambda \in[0,1] .
$$

For $K, L, r \geq 0$ constants with $C$ and $\psi$ as above, let

$$
C_{K}=\{y \in C:\|y\|<K\}
$$

and

$$
C(\psi, L, K)=\{y \in C: \psi(y) \geq L \text { and }\|y\| \leq K\} .
$$

Our consideration is based on the following fixed point theorem given by Leggett and Williams in 1979 [19] (see also Guo and Lakshmikantham [11]).

Theorem 2.1. Let $E$ be a Banach space, $C \subset E$ a cone in $E$ and $R>0$ a constant. Suppose there exists a concave positive continuous functional on $C$ with $\psi(y) \leq\|y\|$ for all $y \in \overline{C_{R}}$ and let $N: \overline{C_{R}} \rightarrow \overline{C_{R}}$ be a continuous compact map. Assume that there are numbers $r, L$ and $K$ with $0<r<L<K \leq R$ such that

$\left(A_{1}\right)\{y \in C(\psi, L, K): \psi(y)>L,\|y\| \leq K\} \neq \varnothing$ and $\psi(N(y))>L \quad$ for all $y \in C(\psi, L, K)$;

$\left(A_{2}\right)\|N(y)\|<r$ for all $y \in \overline{C_{r}}$;

$\left(A_{3}\right) \psi(N(y))>L$ for all $y \in C(\psi, L, R)$ with $\|N(y)\|>K$.

Then $N$ has at least three fixed points $y_{1}, y_{2}, y_{3}$ in $\overline{C_{R}}$. Furthermore, we have

$$
y_{1} \in C_{r}, \quad y_{2} \in\{y \in C(\psi, L, R): \psi(y)>L\}
$$

and

$$
y_{3} \in \overline{C_{R}}-\left\{C(\psi, L, R) \cup \overline{C_{r}}\right\} .
$$


Vol. LIII (2015) Existence of three positive solutions for boundary value problem 61

\section{Main results}

Let us start by defining what we mean by a solution of the problem (1.1)(1.3).

Set

$$
\mathcal{B}_{1}=\left\{y:(-\infty, 1] \rightarrow R:\left.y\right|_{(-\infty, 0]} \in \mathcal{B},\left.y\right|_{J} \in C^{2}(J, R)\right\},
$$

and let $\|\cdot\|_{1}$ the seminorm in $\mathcal{B}_{1}$ defined by :

$$
\|y\|_{1}=\left\|y_{0}\right\|_{\mathcal{B}}+\sup \{|y(t)|: 0 \leq t \leq 1\}, y \in \mathcal{B}_{1} .
$$

Definition 3.1. A function $y \in \mathcal{B}_{1}$ is said to be a solution of (1.1)-(1.3) if $y$ satisfies the equation $D^{\alpha} y(t)=f\left(t, y_{t}\right)$ on $J$, and conditions $y(t)=\phi(t), t \in$ $(-\infty, 0], y(0)=y^{\prime}(0)=0, \beta y(\eta)=y(1)$.

For the existence of solutions for the problem (1.1) - (1.3), we need the following auxiliary Lemmas.

Lemma 3.1. [28] Let $\alpha>0$, then the differential equation

$$
D^{\alpha} h(t)=0
$$

has solutions $h(t)=c_{1} t^{\alpha-1}+c_{2} t^{\alpha-22}+\ldots .+c_{n} t^{\alpha-n}, c_{i} \in R, \quad i=1,2, \ldots ., n$, $n=[\alpha]+1$.

Lemma 3.2. [28] Let $\alpha>0$, then

$$
I^{\alpha} D^{\alpha} h(t)=h(t)+c_{1} t^{\alpha-1}+c_{2} t^{\alpha-22}+\ldots .+c_{n} t^{\alpha-n}
$$

for some $c_{i} \in R, \quad i=0,1,2, \ldots, n-1, n=[\alpha]+1$.

Let $h$ be a continuous function and consider the boundary value problem problem

$$
\begin{gathered}
D^{\alpha} y(t)=\rho(t), \text { a.e }, t \in J=[0,1] \\
y(0)=y^{\prime}(0)=0, \beta y(\eta)=y(1), \\
y(t)=\phi(t), \quad t \in(-\infty, 0] .
\end{gathered}
$$

Lemma 3.3. The problem (3.1) - (3.3) has a unique solution given by:

$$
y(t)=\int_{0}^{1} G(t, s) \rho(s) d s+\frac{\beta t^{\alpha-1}}{1-\beta \eta} \int_{0}^{1} G(\eta, s) \rho(s) d s,
$$

where $G(t, s)$ is given by :

$$
G(t, s)=\frac{1}{\Gamma(\alpha)} \begin{cases}t^{\alpha-1}(1-s)^{\alpha-1}-(t-s)^{\alpha-1}, & 0 \leq s \leq t \leq 1 \\ t^{\alpha-1}(1-s)^{\alpha-1}, & 0 \leq t \leq s \leq 1\end{cases}
$$


By Lemma 3.2 we have $y(t)=c_{1} t^{\alpha-1}+c_{2} t^{\alpha-2}-\frac{1}{\Gamma(\alpha)} \int_{0}^{t}(t-s)^{\alpha-1} \sigma(s) d s$, in view that $y(0)=0$ we have $c_{2}=0$ and then

$$
y(t)=c_{1} t^{\alpha-1}-\frac{1}{\Gamma(\alpha)} \int_{0}^{t}(t-s)^{\alpha-1} \rho(s) d s
$$

$y(1)=c_{1}-\frac{1}{\Gamma(\alpha)} \int_{0}^{1}(1-s)^{\alpha-1} \rho(s) d s$

$\beta y(\eta)=\beta \eta^{\alpha-1} c_{1}-\frac{\beta}{\Gamma(\alpha)} \int_{0}^{\eta}(\eta-s)^{\alpha-1} \rho(s) d s$,

and so :

$$
\begin{aligned}
y(t) & =\frac{1}{\Gamma(\alpha)} \int_{0}^{1}(1-s)^{\alpha-1} t^{\alpha-1} \rho(s) d s \\
& +\frac{\beta \eta^{\alpha-1}}{\left(1-\beta \eta^{\alpha-1}\right) \Gamma(\alpha)} \int_{0}^{1}(1-s)^{\alpha-1} t^{\alpha-1} \rho(s) d s \\
& -\frac{\beta}{\left(1-\beta \eta^{\alpha-1}\right) \Gamma(\alpha)} \int_{0}^{\eta}(\eta-s)^{\alpha-1} t^{\alpha-1} \rho(s) d s \\
& \quad-\frac{1}{\Gamma(\alpha)} \int_{0}^{t}(t-s)^{\alpha-1} \rho(s) d s, \\
y(t)= & \frac{1}{\Gamma(\alpha)} \int_{0}^{1}\left[(1-s)^{\alpha-1} t^{\alpha-1}-(t-s)^{\alpha-1}\right] \rho(s) d s \\
+ & \frac{1}{\Gamma(\alpha)} \int_{t}^{1}(1-s)^{\alpha-1} t^{\alpha-1} \rho(s) d s \\
+ & \frac{\beta t^{\alpha-1}}{\left.1-\beta \eta^{\alpha-1}\right) \Gamma(\alpha)}\left(\int_{0}^{\eta}\left[(1-s)^{\alpha-1} \eta^{\alpha-1}-(\eta-s)^{\alpha-1}\right] \rho(s) d s\right. \\
+ & \left.\int_{\eta}^{1}(1-s)^{\alpha-1} \eta^{\alpha-1} \rho(s) d s\right) .
\end{aligned}
$$

And then we have:

$$
y(t)=\int_{0}^{1} G(t, s) \rho(s) d s+\frac{\beta t^{\alpha-1}}{1-\beta \eta^{\alpha-1}} \int_{0}^{1} G(\eta, s) \rho(s) d s .
$$

By an easy computation we obtain (3.4) :

Lemma 3.4. the function defined by (3.5) satisfies the following conditions:

(i) $G(t, s) \geq 0 \quad G(t, s) \leq G(s, s)$, for all $s, t \in[0,1]$ 
Vol. LIII (2015) Existence of three positive solutions for boundary value problem 63

(ii) $\max _{0 \leq t \leq b} \int_{0}^{1} G(t, s) d s=\frac{\Gamma(\alpha)}{\Gamma(2 \alpha)}$.

(iii) there exists a positive function $g \in C(0,1)$ such that $\min _{\gamma \leq t \leq \delta} G(t, s) \geq g(s) G(s, s), s \in(0,1)$, where $0<\gamma<\delta<1$ and

$$
g(s)= \begin{cases}\frac{\delta^{\alpha-1}(1-s)^{\alpha-1}-(\delta-s)^{\alpha-1}}{t^{\alpha-1}(1-s)^{\alpha-1}}, & \text { if } s \in\left[0, m_{1}\right], \\ \left(\frac{\gamma}{s}\right)^{\alpha-1}, & \text { if. } s \in\left[m_{1}, 1\right],\end{cases}
$$

Where $\gamma<m_{1}<\delta$.

Proof. By definition of $G(t, s)$, for all $(t, s) \in[0,1] \times[0,1]$ if $s \leq t$ it can be written :

$$
\begin{aligned}
G(t, s) & =\frac{1}{\Gamma(\alpha)}\left(t^{\alpha-1}(1-s)^{\alpha-1}-(t-s)^{\alpha-1}\right) \\
& \geq \frac{1}{\Gamma(\alpha)}\left[t^{\alpha-1}(1-s)^{\alpha-1}-(t-t s)^{\alpha-1}\right] \\
& \geq \frac{t^{\alpha-1}}{\Gamma(\alpha)} t^{\alpha-1}\left[(1-s)^{\alpha-1}-(1-s)^{\alpha-1}\right]=0
\end{aligned}
$$

and if $t \leq s$ it is obvious that $G(t, s) \geq 0$ Therefore one can conclude that: $G(t, s) \geq 0$, for all $(t, s) \in[0,1] \times[0,1]$.

Let $L(t, s)=t^{\alpha-1}(1-s)^{\alpha-1}-(t-s)^{\alpha-1}$ for $0 \leq s \leq t \leq 1$. Then

$$
\begin{aligned}
\frac{d L(t, s)}{d t} & =(\alpha-1)\left[t^{\alpha-2}(1-s)^{\alpha-1}-(t-s)^{\alpha-2}\right] \\
& =(\alpha-1) t^{\alpha-2}\left[t^{\alpha-2}(1-s)^{\alpha-1}-\left(1-\frac{s}{t}\right)^{\alpha-2}\right] \\
& \leq(\alpha-1) t^{\alpha-2}\left[(1-s)^{\alpha-1}-(1-s)^{\alpha-2}\right] \leq 0
\end{aligned}
$$

which implies that $L(t, s)$ is non-increasing for all $s \in[0,1]$, hence, we obtain that :

$$
L(t, s) \leq L(s, s) \text { for all } 0 \leq s \leq t \leq 1 .
$$

Thus, by definition of $G$ and (3.7), we Know that $G(t, s) \leq G(s, s)$ for all $s, t \in[0,1]$.

(ii) Let $J(t, s)=t^{\alpha-1}(1-s)^{\alpha-1}$, for $t \leq s \leq b$, since $L(t, s)$ is non increasing, $J(t, s)$ is non decreasing for all $s \in[0,1]$ then one can give : 


$$
\begin{aligned}
\min _{\gamma \leq t \leq \delta} G(t, s) & =\frac{1}{\Gamma(\alpha)} \begin{cases}L(\gamma, s), & s \in[0, \gamma] \\
\min \{L(\gamma, s), J(\delta, s)\} & s \in[\gamma, \delta] \\
J(\delta, s), & s \in[\delta, s] .\end{cases} \\
& =\frac{1}{\Gamma(\alpha)} \begin{cases}L(\gamma, s), \quad s \in\left[0, m_{1}\right] \\
J(\delta, s), \quad s \in\left[m_{1}, 1\right]\end{cases} \\
& =\frac{1}{\Gamma(\alpha)} \begin{cases}\left(\gamma^{\alpha-1}(1-s)^{\alpha-1}-(\gamma-s)^{\alpha-1}\right), \\
s \in\left[0, m_{1}\right] \\
\delta^{\alpha-1}(1-s)^{\alpha-1}, & s \in\left[m_{1}, 1\right]\end{cases}
\end{aligned}
$$

where $\gamma \leq m_{1} \leq \delta$ is the solution of equation :

$$
\gamma^{\alpha-1}(1-s)^{\alpha-1}-(\gamma-s)^{\alpha-1}=\delta^{\alpha-1}(1-s)^{\alpha-1} .
$$

It follows from the monotonocity of $L$ and $J$ that:

$$
\max _{t \leq 0 \leq b} G(t, s)=G(s, s)=\frac{s^{\alpha-1}(1-s)^{\alpha-1}}{\Gamma(\alpha)} .
$$

Thus we set $g(s)$ as in (3.6).

By (3.8) and beta function we have :

$$
\max _{0 \leq t \leq 1} \int_{0}^{1} G(t, s) d s=\frac{\Gamma(\alpha)}{\Gamma(2 \alpha)} .
$$

Therefore the proof is complete.

We define now :

$$
\mu=\min _{t \in[\gamma, \delta]} g(t), \sigma=\max \left\{\gamma^{\alpha-1}, \mu\right\} .
$$

For the next theorem we need the following assumptions:

$\left(H_{1}\right) f$ is a continuous function.

$\left(H_{2}\right)$ There exist a functions $q:[0, \infty) \rightarrow[0, \infty)$ continuous and nondecreasing, a function $h:[0, \infty) \rightarrow[0, \infty)$ continuous and nonincreasing, $p_{1} \in C\left(J, R_{+}\right), \quad p_{2} \in C\left(J, R_{+}\right)$such that

$$
p_{2}(t) h(\|u\|) \leq f(t, u) \leq p_{1}(t) q(\|u\|),
$$

for each $(t, u) \in J \times \mathcal{B}$. 
Vol. LIII (2015) Existence of three positive solutions for boundary value problem 65

$\left(H_{3}\right)$ There exists a constant $r>0$ such that:

$$
q\left(K r+M\|\phi\|_{\mathcal{B}}\right)\left\|p_{1}\right\|_{\infty} \frac{\Gamma(\alpha)}{\Gamma(2 \alpha)}\left\{1+\frac{\beta}{1-\beta \eta^{\alpha-1}}\right\} \leq r .
$$

$\left(H_{4}\right)$ There exists a constant $L>r$ such that

$$
h\left(K L+M\|\phi\|_{\mathcal{B}}\right)\left\|p_{2}\right\|_{\infty}\left\{\int_{\gamma}^{\delta} G(t, s) d s+\frac{\beta}{1-\beta \eta^{\alpha-1}} \int_{\gamma}^{\delta} G(\eta, s) d s\right\} \geq L .
$$

$\left(H_{5}\right)$ There exist $0<r<L \leq \sigma R$ such that

$$
q\left(K R+M\|\phi\|_{\mathcal{B}}\right)\left\|p_{1}\right\|_{\infty} \frac{\Gamma(\alpha)}{\Gamma(2 \alpha)}\left\{1+\frac{\beta}{1-\beta \eta^{\alpha-1}}\right\} \leq R .
$$

Theorem 3.5. Assume (H1)-(H5) are satisfied. Then problem (1.1)-(1.3) has at least three positives solutions.

Proof. Our result is based on Legett-Williams fixed point Theorem. Transform the problem (1.1)-(1.3) into a fixed point problem. Consider the operator

$$
N: \mathcal{B}_{1} \rightarrow \mathcal{B}_{1}
$$

defined by:

$N(y)(t)=\left\{\begin{array}{lr}\phi(t), & t \in(-\infty, 0], \\ \int_{0}^{1} G(t, s) f\left(s, y_{s}\right) d s+\frac{\beta t^{\alpha-1}}{1-\beta \eta^{\alpha-1}} \int_{0}^{1} G(\eta, s) f\left(s, y_{s}\right) d s, & t \in[0,1],\end{array}\right.$

and the function $G(t, s)$ is given by (3.5). Clearly, the fixed points of the operator $N$ are solution of the problem (1.1)-(1.3).

Let $x():.(-\infty, 1] \rightarrow R$ be the function defined by :

$$
x(t)= \begin{cases}\phi(t), & \text { if } t \in(-\infty, 0], \\ 0, & \text { if } t \in[0,1] .\end{cases}
$$

Then $x_{0}=\phi$. For each $z \in \mathcal{B}$ with $z_{0}=0$, we denote by $\bar{z}$ the function defined by

$$
\bar{z}(t)= \begin{cases}0, & \text { if } t \in(-\infty, 0], \\ z(t), & \text { if } t \in[0,1] .\end{cases}
$$

If $y(\cdot)$ satisfies the integral equation :

$$
y(t)=\int_{0}^{1} G(t, s) f\left(s, y_{s}\right) d s+\frac{\beta t^{\alpha-1}}{1-\beta \eta^{\alpha-1}} \int_{0}^{1} G(\eta, s) f\left(s, y_{s}\right) d s,
$$


we can decompose $y($.$) into y(t)=\bar{z}(t)+x(t), 0 \leq t \leq 1$, which implies $y_{t}=\bar{z}_{t}+x_{t}$, for every $t \in[0,1]$, and the function $z(\cdot)$ satisfies :

$$
\left.z(t)=\int_{0}^{1} G(t, s) f\left(s, \bar{z}_{s}+x_{s}\right)\right)+\frac{\beta t^{\alpha-1}}{1-\beta \eta^{\alpha-1}} \int_{0}^{1} G(\eta, s) f\left(s, y_{s}\right) d s .
$$

Set

$$
\mathcal{B}_{0}=\left\{z \in C([0,1], R): z_{0}=0\right\} .
$$

Let $\|.\|_{1}$ be the seminorm in $\mathcal{B}_{0}$ defined by:

$$
\left.\|z\|_{1}=\left\|z_{0}\right\|_{\mathcal{B}}+\sup \{|z(s)|: 0 \leq s \leq 1\}=\sup \{|z(s)|: 0 \leq s \leq 1\}\right)=\|z\|_{0} .
$$

$\mathcal{B}_{0}$ is a Banach space with the norm $\|.\|_{0}$. We define the operator $P: \mathcal{B}_{0} \rightarrow \mathcal{B}_{0}$ by :

$$
P(z)(t)=\int_{0}^{1} G(t, s) f\left(s, \bar{z}_{s}+x_{s}\right) d s+\frac{\beta t^{\alpha-1}}{1-\beta \eta^{\alpha-1}} \int_{0}^{1} G(\eta, s) f\left(s, \bar{z}_{s}+x_{s}\right) d s .
$$

Obviously the operator $N$ has a fixed point is equivalent to $P$ has one, so we need to prove that $P$ has a fixed point.

We show firstly that $P$ is completely continuous :

Step 1: $P$ is continuous

Let $\left\{z_{n}\right\}$ be a sequence such that $z_{n} \rightarrow z$ in $\mathcal{B}_{0}$. Then

$$
\begin{aligned}
\left|P\left(z_{n}\right)(t)-P(z)(t)\right| & \\
\quad \leq & \left.\int_{0}^{t} G(t, s) \mid f\left(s, \bar{z}_{n_{s}}+x_{s}\right)\right)-f\left(s, \bar{z}_{s}+x_{s}\right) \mid d s+ \\
& \left.+\frac{\beta}{1-\beta \eta^{\alpha-1}} \int_{0}^{1} G(\eta, s) \mid f\left(s, \bar{z}_{n_{s}}+x_{s}\right)\right)-f\left(s, \bar{z}_{s}+x_{s}\right) \mid d s . \\
& \left.\leq \| f\left(., \bar{z}_{n_{(.)}}+x_{(.)}\right)\right)-f\left(., \bar{z}_{(.)}+x_{(.)}\right) \| \max _{0 \leq t \leq 1} \int_{0}^{1} G(t, s) d s \\
& \left.+\frac{\beta}{1-\beta \eta^{\alpha-1}} \| f\left(., \bar{z}_{n_{(.)}}+x_{(.)}\right)\right)-f\left(., \bar{z}_{(.)}+x_{(.)}\right) \| \max _{0 \leq t \leq 1} \int_{0}^{1} G(\eta, s) d s . \\
& \leq \frac{\Gamma(\alpha)}{\Gamma(2 \alpha)}\left(1+\frac{\beta}{1-\beta \eta^{\alpha-1}}\right)\left(\left\|f\left(., \bar{z}_{n_{(.)}}+x_{(.)}\right)-f\left(., \bar{z}_{(.)}+x_{(.)}\right)\right\|\right) .
\end{aligned}
$$

Using $\left(H_{1}\right)$ we have:

$$
\left\|P\left(z_{n}\right)-P(z)\right\|_{0} \rightarrow 0 \text { as } n \rightarrow \infty .
$$


Vol. LIII (2015) Existence of three positive solutions for boundary value problem 67

Step 2: $P$ maps bounded sets into bounded sets in $\mathcal{B}_{0}$.

Indeed, it is enough to show that for any $\xi>0$, there exists a positive constant $\ell$ such that for each $z \in B_{\xi}=\left\{z \in \mathcal{B}_{0}:\|z\|_{0} \leq \eta^{*}\right\}$, one has $\|P z\|_{\infty} \leq \ell$ by $(H 2)$ we have for each $t \in[0,1]$,

$$
\begin{aligned}
|P(z)(t)| \leq & \int_{0}^{1}\left|G(t, s) f\left(s, \bar{z}_{s}+x_{s}\right)\right| d s+\frac{\beta t^{\alpha-1}}{1-\beta \eta^{\alpha-1}} \\
& \int_{0}^{1}\left|G(\eta, s) f\left(s, \bar{z}_{s}+x_{s}\right)\right| d s \\
& \leq \int_{0}^{1} G(t, s) p_{1}(s) q\left(\left\|\bar{z}_{s}+x_{s}\right\|\right) d s \\
& +\frac{\beta t^{\alpha-1}}{1-\beta \eta^{\alpha-1}} \int_{0}^{1} G(\eta, s) p_{1}(s) q\left(\left\|\bar{z}_{s}+x_{s}\right\|\right) \\
& \leq \frac{\Gamma(\alpha)}{\Gamma(2 \alpha)}\left(1+\frac{\beta}{1-\beta \eta^{\alpha-1}}\right)\left\|p_{1}\right\|_{\infty} q\left(\eta^{*}\right)=: \ell \\
\left\|\bar{z}_{s}+x_{s}\right\|_{\mathcal{B}} \leq & \left\|\bar{z}_{s}\right\|_{\mathcal{B}}+\left\|x_{s}\right\|_{\mathcal{B}} \\
\leq & K(t) \sup \{|z(s)|: 0 \leq s \leq t\}+M(t)\left\|z_{0}\right\|_{\mathcal{B}} \\
& \\
& K(t) \sup \{|x(s)|: 0 \leq s \leq t\}+M(t)\left\|x_{0}\right\|_{\mathcal{B}} \\
\leq & K \sup \{|z(s)|: 0 \leq s \leq t\}+M\|\phi\|_{\mathcal{B}}+K|\phi(0)| . \\
\leq & K \sup \{|z(s)|: 0 \leq s \leq t\}+M\|\phi\|_{\mathcal{B}} . \\
\leq & K \xi+M\|\phi\|_{\mathcal{B}}=\eta * .
\end{aligned}
$$

Step 3: $P$ maps bounded sets into equicontinuous sets of $\mathcal{B}_{0}$.

Let $\tau_{1}, \tau_{2} \in[0,1], \tau_{1}<\tau_{2}$, let $B_{\xi}$ a bounded set of $\mathcal{B}_{0}$ as in Step 2, and let $z \in B_{\xi}$. Then,

$$
\begin{aligned}
\mid P(z)\left(\tau_{2}\right)- & P(z)\left(\tau_{1}\right) \mid \leq \\
& \leq \mid \int_{0}^{1} G\left(\tau_{2}, s\right) f\left(s, \bar{z}_{s}+x_{s}\right) d s+\frac{\beta \tau_{2}^{\alpha-1}}{1-\beta \eta^{\alpha-1}} \int_{0}^{1} G(\eta, s) f\left(\bar{z}_{s}+x_{s}\right) d s \\
& -\int_{0}^{1} G\left(\tau_{1}, s\right) f\left(s, \bar{z}_{s}+x_{s}\right) d s\left|-\frac{\beta \tau_{1}^{\alpha-1}}{1-\beta \eta^{\alpha-1}} \int_{0}^{1} G(\eta, s) f\left(\bar{z}_{s}+x_{s}\right) d s\right| \\
& \leq\left|\int_{0}^{1} G\left(\tau_{2}, s\right) f\left(s, \bar{z}_{s}+x_{s}\right) d s-\int_{0}^{1} G\left(\tau_{1}, s\right) f\left(s, \bar{z}_{s}+x_{s}\right) d s\right| \\
& +\beta \frac{\tau_{2}^{\alpha-1}-\tau_{1}^{\alpha-1}}{1-\beta \eta^{\alpha-1}} \int_{0}^{1} G(\eta, s)\left|f\left(\bar{z}_{s}+x_{s}\right)\right| d s \\
& \leq \int_{0}^{1}\left|G\left(\tau_{2}, s\right)-G\left(\tau_{1}, s\right)\right| p_{1}(s) q\left(\left\|\bar{z}_{s}+x_{s}\right\|_{\mathcal{B}}\right) d s . \\
& +\beta \frac{\tau_{2}^{\alpha-1}-\tau_{1}^{\alpha-1}}{1-\beta \eta^{\alpha-1}} \int_{0}^{1} G(\eta, s) p(s) q\left(\left\|\bar{z}_{s}+x_{s}\right\|_{\mathcal{B}}\right) d s \\
& \leq\left\|p_{1}\right\|_{\infty} q\left(\eta^{*}\right) \max _{s \in[0,1]}\left(\left|G\left(\tau_{1}, s\right)-G\left(\tau_{2}, s\right)\right|\right) \\
& +\beta \frac{\tau_{2}^{\alpha-1}-\tau_{1}^{\alpha-1}}{1-\beta \eta^{\alpha-1}}\|p\|_{\infty} q\left(\eta^{*}\right) \max _{s \in[0,1]}(|G(\eta, s)| .)
\end{aligned}
$$


Since $G$ is continuous function we have:

$$
\begin{aligned}
\left|P(z)\left(\tau_{2}\right)-P(z)\left(\tau_{1}\right)\right| & \leq\left\|p_{1}\right\|_{\infty} q\left(\eta^{*}\right)\left[\max _{s \in[0,1]}\left(\left|G\left(\tau_{1}, s\right)-G\left(\tau_{2}, s\right)\right|\right)\right. \\
& \left.+\beta \frac{\tau_{2}^{\alpha-1}-\tau_{1}^{\alpha-1}}{1-\beta \eta^{\alpha-1}} \max _{s \in[0,1]}(\mid G(\eta, s)) \mid .\right]
\end{aligned}
$$

Since $G$ is continuous function we have: The right-hand side of the above inequality tends to zero, as $t_{2} \rightarrow t_{1}$ and this proves that: $P(B(0, \xi))$ is equicontinuous in $\mathcal{B}_{0}$. As a consequence of the steps 1 to 3 together with the Ascoli-Arzela Theorem, we can conclude that the operator $P: \mathcal{B}_{0} \rightarrow \mathcal{B}_{0}$ is completely continuous.

Let

$$
\mathcal{C}=\left\{z \in \mathcal{B}_{0}: z(t) \geq 0 \min _{t \in[\gamma, \delta]} z(t) \geq \frac{\sigma}{3}\|z\|_{0} \text { for } t \in J\right\}
$$

be a cone in $\mathcal{B}_{0}$.

We prove now that $P: \mathcal{C} \rightarrow \mathcal{C}$ is well defined. Let $z \in \mathcal{C}$, then it follows from Lemma (3.4), (3.9), (3.10) that:

$$
\begin{aligned}
\|P z\|_{0} & \leq \int_{0}^{1} G(s, s) f\left(s, \bar{z}_{s}+x_{s}\right) d s+ \\
& +\frac{\beta t^{\alpha-1}}{1-\beta \eta^{\alpha-1}} \int_{0}^{1} G(s, s) f\left(s, \bar{z}_{s}+x_{s}\right) d s \\
& =\left(\int_{0}^{\gamma}+\int_{\gamma}^{\delta}+\int_{\delta}^{1}\right) G(s, s) f\left(\bar{z}_{s}+x_{s}\right) d s \\
& +\frac{\beta t^{\alpha-1}}{1-\beta \eta^{\alpha-1}}\left(\int_{0}^{\gamma}+\int_{\gamma}^{\delta}+\int_{\delta}^{1}\right) G(s, s) f\left(\bar{z}_{s}+x_{s}\right) d s \\
& \leq 3\left[\int_{\gamma}^{\delta} G(s, s) f\left(\bar{z}_{s}+x_{s}\right) d s+\right. \\
& \left.+\frac{\beta}{1-\beta \eta^{\alpha-1}} \int_{\gamma}^{\delta} G(s, s) f\left(\bar{z}_{s}+x_{s}\right) d s .\right]
\end{aligned}
$$

On the other hand lemma 3.4 and (3.9) imply that for any $t \in[\gamma, \delta]$ 
Vol. LIII (2015) Existence of three positive solutions for boundary value problem 69

$$
\begin{aligned}
&(P z)(t)=\int_{0}^{1} G(t, s) f\left(s, \bar{z}_{s}+x_{s}\right) d s+ \\
&+\frac{\beta t^{\alpha-1}}{1-\beta \eta^{\alpha-1}} \int_{0}^{1} G(\eta, s) f\left(s, \bar{z}_{s}+x_{s}\right) d s \\
& \geq \int_{\gamma}^{\delta} G(t, s) f\left(s, \bar{z}_{s}+x_{s}\right) d s+ \\
&+\frac{\beta t^{\alpha-1}}{1-\beta \eta^{\alpha-1}} \int_{\gamma}^{\delta} G(\eta, s) f\left(s, \bar{z}_{s}+x_{s}\right) d s \\
&\left.\geq \int_{\gamma}^{\delta} g(s)\right) G(s, s) f\left(s, \bar{z}_{s}+x_{s}\right) d s+ \\
&+\frac{\beta \gamma^{\alpha-1}}{1-\beta \eta^{\alpha-1}} \int_{\gamma}^{\delta} g(s) G(s, s) f\left(s, \bar{z}_{s}+x_{s}\right) d s \\
& \geq \sigma\left[\int_{\gamma}^{\delta} G(s, s) f\left(s, \bar{z}_{s}+x_{s}\right) d s+\frac{\beta}{1-\beta \eta^{\alpha-1}}\right. \\
&\left.\times \quad \int_{\gamma}^{\delta} G(s, s) f\left(s, \bar{z}_{s}+x_{s}\right) d s\right] . \\
& \geq \frac{\sigma}{3}\|P z\|_{0} .
\end{aligned}
$$

This fact directly implies that $P: \mathcal{C} \rightarrow \mathcal{C}$ is well defined.

Using the hypotheses $\left(H_{1}\right)-\left(H_{2}\right)$ and $\left(H_{5}\right) P: \overline{C_{R}} \rightarrow \overline{C_{R}}$ is well defined and completely continuous.

Let $\psi: \mathcal{C} \rightarrow[0, \infty)$ be defined by :

$$
\psi(z)=\min _{t \in[\gamma, \delta]} z(t)
$$

It is clear that $\psi$ is a nonnegative concave continuous functional and

$$
\psi(z) \leq\|z\|_{0} \quad \text { for } z \in \overline{C_{R}}
$$

Now it remains to show that the hypotheses of Theorem 2.1 are satisfied. First notice that condition $\left(A_{2}\right)$ of Theorem 2.1 holds since for $z \in \overline{C_{r}}$, and 
from $\left(H_{2}\right),\left(H_{3}\right)$ and $(3.11)$ we have :

$$
\begin{gathered}
\|P(z)\|=\max _{0 \leq t \leq 1}|P(z)(t)| \\
=\max _{0 \leq t \leq 1} \mid\left(\int_{0}^{1} G(t, s) f\left(s, \bar{z}_{s}+x_{s}\right) d s+\right. \\
\left.+\quad \frac{\beta t^{\alpha-1}}{1-\beta \eta^{\alpha-1}} \int_{0}^{1} G(\eta, s) f\left(s, \bar{z}_{s}+x_{s}\right) d s \mid\right) \\
\leq \quad \max _{0 \leq t \leq 1}\left(\int_{0}^{1}|G(t, s)| q\left(\left\|\bar{z}_{s}+x_{s}\right\|\right)\left|p_{1}(s)\right| d s+\right. \\
\left.+\quad \frac{\beta}{1-\beta \eta^{\alpha-1}} \int_{0}^{1}|G(\eta, s)| q\left(\left\|\bar{z}_{s}+x_{s}\right\|\right)\left|p_{1}(s)\right|\right) \\
\leq \quad \max _{0 \leq t \leq 1}\left(\int_{0}^{1} G(t, s) q\left(K\|z\|_{0}+M\|\phi\|_{\mathcal{B}}\right) p_{1}(s) d s\right. \\
\left.+\quad \frac{\beta}{1-\beta \eta^{\alpha-1}} \int_{0}^{1} G(\eta, s) q\left(K\|z\|_{0}+M\|\phi\|_{\mathcal{B}}\right) p_{1}(s) d s\right) \\
\leq \quad q\left(K r+M\|\phi\|_{\mathcal{B}}\right)\left\|p_{1}\right\|_{\infty} \max _{0 \leq t \leq 1}\left\{\int_{0}^{1} G(t, s) d s+\right. \\
\left.+\quad \frac{\beta}{1-\beta \eta^{\alpha-1}} \int_{0}^{1} G(\eta, s) d s\right\} \\
\leq \quad q\left(K r+M\|\phi\|_{\mathcal{B}}\right)\left\|p_{1}\right\|_{\infty} \frac{\Gamma(\alpha)}{\Gamma(2 \alpha)}\left\{1+\frac{\beta}{1-\beta \eta^{\alpha-1}}\right\} \\
\leq r .
\end{gathered}
$$

We show now that that condition $\left(A_{1}\right)$ of Theorem is satisfied.

Clearly if $z \in C\left(\psi, L, \frac{L}{\sigma}\right)$ then $L \leq z(s) \leq \frac{L}{\sigma}, s \in[\gamma, \delta]$. and then $\left\{z \in C\left(\psi, L, \frac{L}{\sigma}\right), \psi(z)>L\right\} \neq \oslash$.

By condition $\left(H_{4}\right)$ we get :

$$
\begin{aligned}
\psi(P(z)) & =\min _{\gamma \leq t \leq \delta}\left(\int_{0}^{1} G(t, s) f\left(s, \bar{z}+x_{s}\right) d s+\right. \\
& \left.+\frac{\beta t^{\alpha-1}}{1-\beta \eta^{\alpha-1}} \int_{0}^{1} G(\eta, s) f\left(s, \bar{z}+x_{s}\right) d s\right) \\
& \geq \min _{\gamma \leq t \leq \delta}\left[\int_{0}^{1} G(t, s) h\left(\left\|\overline{z_{s}}+x_{s}\right\|\right) p_{2}(s) d s+\right. \\
& \left.+\frac{\beta t^{\alpha-1}}{1-\beta \eta^{\alpha-1}} \int_{0}^{1} G(\eta, s) h\left(\left\|\overline{z_{s}}+x_{s}\right\|\right) p_{2}(s) d s\right]
\end{aligned}
$$


Vol. LIII (2015) Existence of three positive solutions for boundary value problem 71

$$
\begin{aligned}
& \geq h\left(K L+M\|\phi\|_{\mathcal{B}}\right)\left\|p_{2}\right\|_{\infty} \min _{\gamma \leq t \leq \delta}\left\{\int_{\gamma}^{\delta} G(t, s) d s+\right. \\
& \left.+\frac{\beta}{1-\beta \eta^{\alpha-1}} \int_{\gamma}^{\delta} G(\eta, s) d s\right\} \geq L .
\end{aligned}
$$

Therefore the condition $\left(A_{1}\right)$ of Theorem 2.1 is satisfied.

Finally, We show that that condition $\left(A_{3}\right)$ of Theorem is also satisfied. If $z \in C(\psi, L, R)$. and $\|P z\|>\frac{L}{\sigma}$,

$$
\begin{aligned}
\psi(P z) & =\min _{t \in[\gamma, \delta]}\left(\int_{0}^{1} G(t, s) f\left(s, \overline{z_{s}}+x_{s}\right) d s+\right. \\
& \left.+\frac{\beta t^{\alpha-1}}{1-\beta \eta^{\alpha-1}} \int_{0}^{1} G(\eta, s) f\left(s, \overline{z_{s}}+x_{s}\right) d s\right) \\
& \geq \sigma\|P z\| \geq L .
\end{aligned}
$$

Thus condition $\left(A_{3}\right)$ holds. Then Leggett and Williams fixed point Theorem implies that $N$ has at least three fixed points $z_{1}, z_{2}, z_{3}$ which are solutions to problem (1.1)-(1.3). Furthermore, we have

$z_{1} \in C_{r}, \quad z_{2} \in\{z \in C(\psi, L, R): \psi(z)>L\}, \quad z_{3} \in C_{R}-\left\{(\psi, L, R) \cup\left(C_{r}\right)\right\}$.

Therefore the condition $\left(A_{3}\right)$ of Theorem is also satisfied.

By Theorem 2.1, there exist three positive solutions $z_{1}, z_{2}, z_{3}$ such that $\left\|z_{1}\right\|<$ $r, L<\alpha\left(z_{2}(t)\right)$, and $\left\|z_{3}\right\|>r$, with $\alpha\left(z_{3}(t)\right)<L$.

Finally the problem $(1.1)-(1.3)$ has three positive solutions $y_{1}, y_{2}, y_{3}$ such that :

$$
y_{i}(t)= \begin{cases}\phi(t), & \text { if } t \in(-\infty, 0], \\ z_{i}(t), & \text { if } t \in[0,1],\end{cases}
$$

$i \in\{1,2,3\}$ therefore, we have the conclusion.

Example 3.1. In this section we give an illustrative example showing the usefulness of our main results. Let us consider the functional differential equation.

$$
\begin{gathered}
D^{\alpha} y(t)=\frac{\left(\left\|y_{t}\right\|+1\right) e^{\frac{t}{100}}}{(1+t) e^{\left\|y_{t}\right\|}}, \quad \text { if } \quad t \in J=[0,1], \alpha=\frac{29}{10}, \\
y(t)=\phi(t) \quad \text { if } t \in(-\infty, 0] \\
y(0)=y^{\prime}(0)=0, \quad y(1)=\frac{3}{4} y\left(\frac{1}{3}\right)=0 .
\end{gathered}
$$


We choose $\phi$ that $\|\phi\|=\frac{1}{10}, \mathcal{B}_{\gamma}$ to be defined by:

$$
\mathcal{B}_{\gamma}=\left\{y \in C((-\infty, 0], R): \lim _{\theta \rightarrow-\infty} e^{\gamma \theta} y(\theta) \text { exists }\right\}
$$

with the norm

$$
\|y\|_{\gamma}=\sup _{\theta \in(-\infty, 0]} e^{\gamma \theta}|y(\theta)| .
$$

Let $y:(-\infty, 1] \rightarrow R$ be such that $y_{0} \in \mathcal{B}_{\gamma}$. Then

$$
\begin{aligned}
\lim _{\theta \rightarrow-\infty} e^{\gamma \theta} y(\theta) & =\lim _{\theta \rightarrow-\infty} e^{\gamma \theta} y(t+\theta) \\
& =\lim _{\theta \rightarrow-\infty} e^{\gamma(\theta-t)} y(\theta) \\
& =e^{\gamma t} \lim _{\theta \rightarrow-\infty} e^{-\gamma \theta} y_{0}(\theta)<+\infty
\end{aligned}
$$

Hence $y_{t} \in \mathcal{B}_{\gamma}$. Finally we prove that

$$
\left\|y_{t}\right\|_{\gamma} \leq K(t) \sup \{|y(s)|: 0 \leq s \leq t\}+M(t)\left\|y_{0}\right\|_{\gamma},
$$

where $K=M=1$ and $H=1$. We have $y(t)=y(t+\phi)$.

If $t+\theta \leq 0$ we get

$$
\left\|y_{t}(\theta)\right\| \leq \sup \{|y(s)|:-\infty \leq s \leq 0\}
$$

For then we have $t+\theta \geq 0$

$$
\left\|y_{t}(\theta)\right\| \leq \sup \{|y(s)|: 0 \leq s \leq t\}
$$

Thus for all $t+\theta \in[0,1]$, we have

$$
\left\|y_{t}(\theta)\right\| \leq \sup \{|y(s)|:-\infty \leq s \leq 0\}+\sup \{|y(s)|: 0 \leq s \leq t\} .
$$

Thus

$$
\left\|y_{t}\right\|_{\gamma} \leq\|y\|_{0}+\sup \{|y(s)|: 0 \leq s \leq t\} .
$$

It is clear that $\left(\mathcal{B}_{\gamma},\|y\|_{\gamma}\right)$ is a Banach space. We can conclude that $\mathcal{B}_{\gamma}$ is a phase space. Set

$$
\begin{gathered}
f(t, u)=\frac{(\|u\|+1) e^{\frac{t}{100}}}{(1+t) e^{\|u\|}}, \quad(t, u) \in J \times \mathcal{B}_{\gamma} . \\
q(u)=u+1, \quad p_{1}(t)=e^{\frac{t}{100}}, \quad u \geq 0, \quad t \in[0,1] \\
h(u)=e^{-u}, \quad p_{2}(t)=\frac{1}{1+t}, \quad u \geq 0, \quad t \in[0,1] .
\end{gathered}
$$


Vol. LIII (2015) Existence of three positive solutions for boundary value problem 73

It follows from the definition of $f, q, p_{1}, h, p_{2}$, that:

$$
p_{2}(t) h(\|u\|) \leq f(t, u) \leq p_{1}(t) q(\|u\|) .
$$

We choose $\gamma=\frac{1}{3}, \quad \delta=\frac{2}{3}$, according to the above $\eta=\frac{1}{3}, \beta=\frac{3}{4}$, then, by direct calculation, we can obtain that

$$
\begin{array}{r}
\min _{\gamma \leq t \leq \delta}\left(\int_{\gamma}^{\delta} G(t, s) d s+\frac{\beta}{1-\beta \eta^{\alpha-1}} \int_{\gamma}^{\delta} G(\eta, s) d s\right)= \\
\min _{\left(\frac{1}{3}\right) \leq t \leq \frac{2}{3}}\left(\int_{\frac{1}{3}}^{\frac{2}{3}} G(t, s) d s+\frac{\frac{3}{4}}{1-\frac{3}{4}\left(\frac{1}{3}\right)^{\frac{19}{10}}} \int_{\frac{1}{3}}^{\frac{2}{3}} G\left(\frac{1}{3}, s\right) d s\right)=0.0831 \\
\sigma=0.121 \\
h\left(K L+M\|\phi\|_{\mathcal{B}}\right)\left\|p_{2}\right\|_{\infty}=h\left(\frac{1}{100}+L\right),
\end{array}
$$

and then :

$$
h\left(K L+M\|\phi\|_{\mathcal{B}}\right)\left\|p_{2}\right\|_{\infty}\left\{\int_{\gamma}^{\delta} G(t, s) d s+\frac{\beta}{1-\beta \eta^{\alpha-1}} \int_{\gamma}^{\delta} G(\eta, s) d s\right\} \geq L,
$$

which yields :

$$
e^{-\frac{1}{100}-L} \times 0.0831 \geq L
$$

Choose $L=0.073$,

in another hand :

$$
q\left(K r+M\|\phi\|_{\mathcal{B}}\right)\left\|p_{1}\right\|_{\infty}=\left(r+1+\frac{1}{100}\right) e^{\frac{1}{100}},
$$

and

$$
\frac{\Gamma\left(\frac{29}{10}\right)}{\Gamma\left(\frac{29}{5}\right)}\left\{1+\frac{\frac{3}{4}}{1-\frac{3}{4}\left(\frac{1}{3}\right)^{\frac{19}{10}}}\right\}=0.0264
$$

and so:

$$
q\left(K r+M\|\phi\|_{\mathcal{B}}\right)\left\|p_{1}\right\|_{\infty} \frac{\Gamma(\alpha)}{\Gamma(2 \alpha)}\left\{1+\frac{\beta}{1-\beta \eta^{\alpha-1}}\right\} \leq r
$$

which yields

$$
\left(r+1+\frac{1}{100}\right) e^{\frac{1}{100}} \times 0.0264 \leq r .
$$


Choose $r=0.070$

$$
\left(R+1+\frac{1}{100}\right) e^{\frac{1}{100}} \times 0.0264 \leq R
$$

Choose $R=10$,

then all assumptions of Theorem 3.5 are satisfied, problem (3.12) - (3.13) has three positive solutions $y_{1}, y_{2}, y_{3}$.

Remark 3.1. Change will occur if we replace the Riemann-Liouville derivative by other kinds of fractional order derivatives, for example if we take Caputo fractional derivative, the operator solution $N$ change in view that lemma 3.2[28] is not the same as Caputo fractional derivative, in another hand the Green function change too, so others hypothesis should be imposed to prove existence result.

\section{Conclusion}

In this paper we have proved the existence of at least three positive solutions for differential equation with fractional order and infinite delay using LeggettWilliams fixed point Theorem.

\section{Acknowledgements}

The author are grateful to the referee for the helpful remarks.

\section{References}

[1] Ravi P. Agarwal, Positive solutions for Dirichlet problems of singular nonlinear fractional differential equations., Journal of Mathem. Anal. and Applications, 371, (2010), 57-58.

[2] Ravi P. Agarwal, Boundary value problems for differential equations involving Riemann-Liouville fractional derivative on the half line., Dynamics of Continuous Discrete and Impulsive System, 18, (2011), 235-244.

[3] Zhanbig Bainov, Positive solutions for boundary value problem of nonlinear fractional differential equation, Journal of Mathematical Anal and Application, 311, (2005), 495-505. 
Vol. LIII (2015) Existence of three positive solutions for boundary value problem 75

[4] Ahmad Bachir, Existence of solutions for irregular boundary value problems of nonlinear fractional differential equations, Applied Mathematics Letters, 23, (2010), 390-394.

[5] Ahmad Bachir, Riemann-Liouville fractional integro-differential equations with fractional nonlocal integral boundary conditions, Boundary Value Problems, 36, (2011), 9 pp.

[6] Jorge Caballero, Positive solutions for a class of singular fractional boundary value problems., Computers Mathematics with Applications, 62, (2011), 1325-1332.

[7] Keller Diethelm, On the solution of nonlinear fractional order differential equations used in the modeling of thermoplasticity, in "Scientific Computing in Chemical Engineering II-Computational Fluid Dynamics,Reaction Engineering and Molecular Properties, Springer-Verlag, (1999), 217-224.

[8] Jiqin Deng, Existence and uniqueness of solutions of initial value problems for nonlinear fractional differential equations, Applied Mathematics Letters, 23, (2010), 676680 .

[9] Ahmad El Sayed, Nonlinear functional differential equations of arbitrary orders, Nonlinear Analysis, 33, (1998), 181-186.

[10] El Sayed Ahmad, Multivalued fractional differential equations, Applied Mathematical Computation, 68, (1995), 15-25.

[11] Dagun Guo, Nonlinear Problems in Abstract Cones, Academic Press, Boston., 1988

[12] Jack Hale, Phase space for retarded equations with infinite delay, Funkcialaj Ekvacioj, 21, (1978), 11-41.

[13] Yoshiyuki Hino, Functional Differential Equations with Infinite Delay, SpringerVerlag, Berlin, 1991.

[14] Franz Kappel, Some considerations to the fundamental theory of infinite delay equations, Journal of Differential Equations, 37, (1980), 141-183.

[15] Ralph Koeller, Application of fractional calculus to the theory of viscolasticity, Journal of Applied Mechanics, 51, (1984), 299-307.

[16] Nickolai Kosmatov, A singular boundary value problem for nonlinear differential equations of fractional order, Journal of Applied Mathematics and Computing, 28, (2009), 125-135.

[17] Anatoly Kilbas, Theory and Applications of Fractional Differential Equations, Elsevier Science B.V., Amsterdam., 2006

[18] vangipuram Lakshmikantham, Theory of Fractional Dynamic Systems, Cambridge Academic Publishers, Cambridge., 2009

[19] Williams Legett, Multiple positive fixed points of nonlinear operators on ordered Banach spaces, Indiana University Mathematics Journal, 428, (1979), 673-688.

[20] Wei Lin, Global existence theory and chaos control of fractional differential equations, Journal of Mathematical Analysis and Applications, 332, (2007), 709-726.

[21] Li Congming, Existence of positive solutions of the boundary value problem for nonlinear fractional differential equations, Computers Mathematics with Applications, 59, (2010), 1363-1375. 
[22] Yang Liu, A sufficient condition for the existence of a positive solution for a nonlinear fractional differential equation with the Riemann Liouville derivative, Applied Mathematics Letters, 25, (2012), 1986-1992.

[23] Kenneth Miller, An Introduction to the Fractional Calculus and Fractional Differential Equations, Wiley, New York., 1993

[24] Zaid Odibat, An algorithm for the numerical solution of differential equations of fractional order, Journal of Applied Mathematics Informatics, 26, (2008), 15-27.

[25] Igor Podlubny, Geometric and physical interpretation of fractional integration and fractional differentiation, Fractional Calculus and Applied Analysis, 5, (2002), 367386.

[26] Tingting Qiu, Existence of positive solutions for singular fractional differential equations, Electronic Journal of Differential Equations, (2008), 1-9.

[27] Konrad Schumacher, Existence and continuous dependence for differential equations with unbounded delay, Archive for Rational Mechanics and Analysis, 64, (1978), 315-335.

[28] Shuqin Zhang, Positive solutions for boundary-value problems of nonlinear fractional diffrential equations, Electron. J. Differential Equations, 36, (2006), 1-12.

[29] Yige Zhao, Positive Solutions to Boundary Value Problems of Nonlinear Fractional Differential Equations, Applied Mathematics and Computation, (2011), 6950-6958.

Hedia Benaouda

Department of Mathematics

University of Tiaret

BP 78 Zaaroura 14000

Tiaret

Algerie

E-mail: b_hedia@univ-tiaret.dz

Received: 25.04.2015

Accepted: 29.06.2015 